\title{
Pulsed neutron fields measurements around a synchrotron storage ring
}

\author{
Marco Caresana ${ }^{1 *}$, Marcello Ballerini ${ }^{2}$, David Garf Ulfbeck ${ }^{4}$, Niels Hertel ${ }^{3}$, Giacomo Paolo Manessi ${ }^{2}$, Carsten \\ Søgaard ${ }^{5}$ \\ ${ }^{1}$ Politecnico di Milano, Department of Energy, Via Lambruschini 4, 20156 Milan, Italy \\ ${ }^{2}$ Else Nuclear srl, Via Tito Vignoli 44, 20146 Milan, Italy \\ ${ }^{3}$ Department of Physics and Astronomy, Institute for Storage Ring Facilities, 8000 Aarhus, Denmark \\ ${ }^{4}$ National Institute for Radiation Protection, 2300 Copenhagen, Denmark \\ 5 eScience group, Niels Bohr Institute, University of Copenhagen, Denmark
}

\begin{abstract}
A measurement campaign was performed for characterizing the neutron ambient dose equivalent, $H^{*}(10)$, in selected positions at ISA, Aarhus, Denmark, around the ASTRID and ASTRID2 storage rings. The neutron stray radiation field is characterized here by very intense radiation bursts with a low repetition rate, which result in a comparatively low average $H^{*}(10)$ rate. As a consequence, devices specifically conceived for operating in pulsed neutron fields must be employed for efficiently measuring in this radiation environment, in order to avoid severe underestimations of the $\mathrm{H}^{*}(10)$ rate. The measurements were performed with the ELSE NUCLEAR LUPIN 5401 BF3-NP rem counter, a detector characterized by an innovative working principle that is not affected by dead time losses. This allowed characterizing both the $\mathrm{H}^{*}(10)$ and the time structure of the radiation field in the pre-selected positions.
\end{abstract}

\section{Introduction}

The need of radiation detectors capable of measuring efficiently in pulsed neutron fields is attracting widespread interest for an increasing number of applications, such as radiation protection monitoring and beam diagnostics around medical and research particle accelerators. Reliable measurements cannot be carried out with conventional rem counters operating in pulse mode, as they underestimate the true neutron interaction rate when exposed to high intensity and short duration radiation bursts. The underestimation is due to dead time losses. Recently [1] it was demonstrated that rem counters operating in pulse mode can properly measure radiation bursts of intensity up to about $10 \mathrm{nSv}\left(\mathrm{H}^{*}(10)\right)$. Above this threshold, the dose underestimation starts to be important and devices specifically conceived for pulsed neutron fields must be used.

An innovative rem counter, called LUPIN (Long interval, Ultra-wide dynamic, PIle-up free, Neutron rem counter) [2,3], has been specifically designed to work in pulsed neutron fields as its working principle is not characterized by any dead time. LUPIN has been employed to characterize the neutron ambient dose equivalent, $H^{*}(10)$, around the Aarhus Storage Ring in Denmark (ASTRID) and ASTRID2 storage rings at the Centre for Storage Ring Facilities (ISA) at Aarhus University, Denmark. The stray radiation field at ISA is characterized, on average, by a low neutron $\mathrm{H}^{*}(10)$ rate.
However, the neutron stray field is generated only during the injection in ASTRID and the extraction phase from ASTRID to ASTRID2, which lasts for a few seconds and happens once every $200 \mathrm{~s}$. This causes a relatively high neutron $\mathrm{H}^{*}(10)$ rate to be measured during the short amount of time.

\section{MATERIALS AND METHODS}

The time structure of the stray neutron field around the ASTRID and ASTRID2 storage rings represents the typical measuring situation for the LUPIN, which has been conceived for coping with very intense radiation bursts. In this paragraph both the experimental facility and the working principle of the LUPIN are discussed.

\subsection{ASTRID and ASTRID2 storage rings}

ASTRID is a $40 \mathrm{~m}$ circumference electron storage ring operating since 1990 . It operates as a $580 \mathrm{MeV}$ electron storage ring, injected from a $100 \mathrm{MeV}$ racetrack microtron. ASTRID2 is a $45.7 \mathrm{~m}$ circumference storage ring located next to ASTRID (see Figure 1) that operates at $580 \mathrm{MeV}$ and is optimised for photon energies from the visible to around $1 \mathrm{keV}[4,5]$.

ASTRID is used as an injector for ASTRID2, which maintains an almost constant circulating current fluctuating around $200 \mathrm{~mA}$. As the current falls below about $150 \mathrm{~mA}$ the beam is injected into ASTRID2 to

* Corresponding author: marco.caresana@polimi.it 
restore the nominal current. The injection occurs every about $150-200 \mathrm{~s}$.

In ASTRID, a fast injection mode can also be operated, with an injection frequency from the microtron of $2 \mathrm{~Hz}$. The neutron stray field around the two storage rings could not be characterised with passive methods (TLDs and CR39) due to the very low expected average $\mathrm{H}^{*}(10)$ rate, which induces very high fluctuations on the results of the readings.

A measurement campaign with the ELSE NUCLEAR LUPIN 5401 BF3-NP rem counter was then planned in agreement with the Danish National Institute for Radiation Protection in order to characterize the neutron $\mathrm{H}^{*}(10)$ rate in 7 selected positions around the storage rings, see Figure 1.

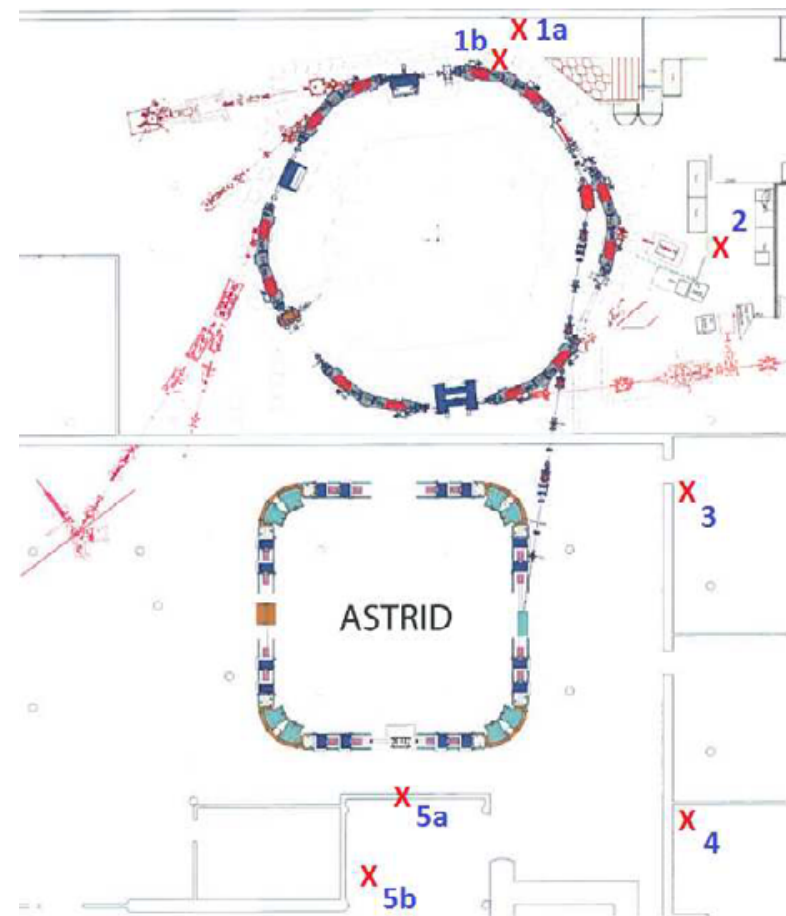

Fig. 1. Map of the measuring positions around the accelerators.

In particular, some of the positions were located in offices and laboratories close to the accelerators and on a platform recently built to allow external visitors to have a look at parts of the ASTRID2 ring. (Position 1b, see Figure 2)

\subsection{LUPIN}

The LUPIN is based on a standard rem counter moderating assembly and is available in two configurations: either a spherical one [2], which embeds a ${ }^{3} \mathrm{He}$ proportional counter, or a cylindrical one [3], which embeds a $\mathrm{BF}_{3}$ proportional counter.

Regardless to the particular configuration, the main difference distinguishing the LUPIN from a standard rem counter is the signal acquisition technique.

It is well known that commercial rem counters can suffer of dead time losses and this effect is particularly severe during an intense and short radiation burst. This effect can lead to important dose underestimation, up to three orders of magnitude [6].
The dead time can be reduced by reducing the shaping time of the output signal, but such an approach can just attenuate the dead time losses, thus reducing the underestimation, but does not fix the problem. Besides there is a physical limit to the reduction of the shaping time defined by the charge mobility inside the proportional counter.

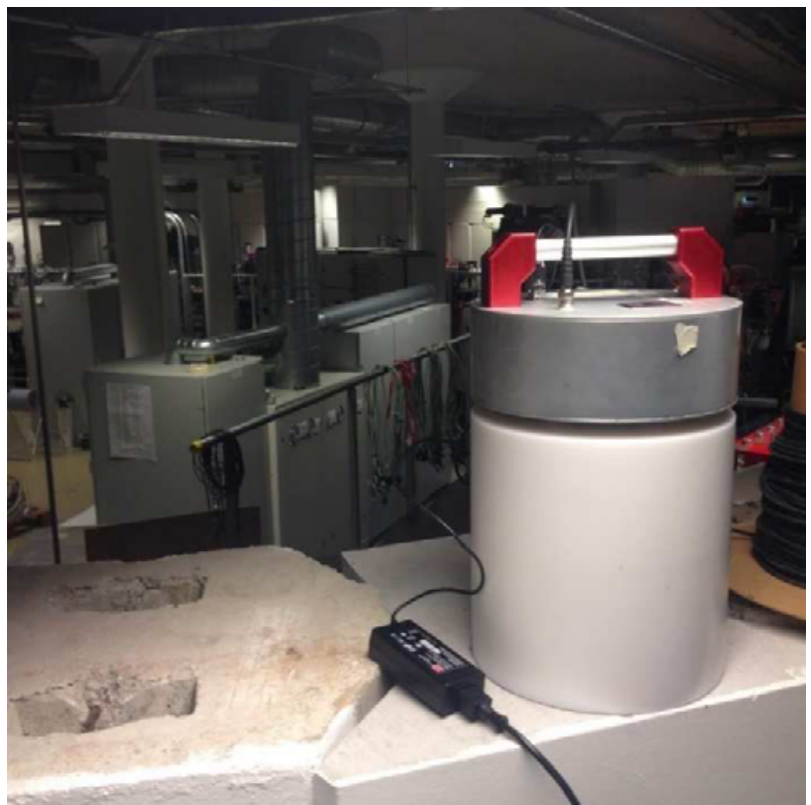

Fig 2. Measuring position $1 \mathrm{~b}$ on top of the visitor platform.

\subsubsection{Working principle of the LUPIN}

The approach adopted in the LUPIN is completely different. The classical acquisition chain composed by preamplifier - amplifier - discriminator - counter is replaced by the electronics shown in figure 3

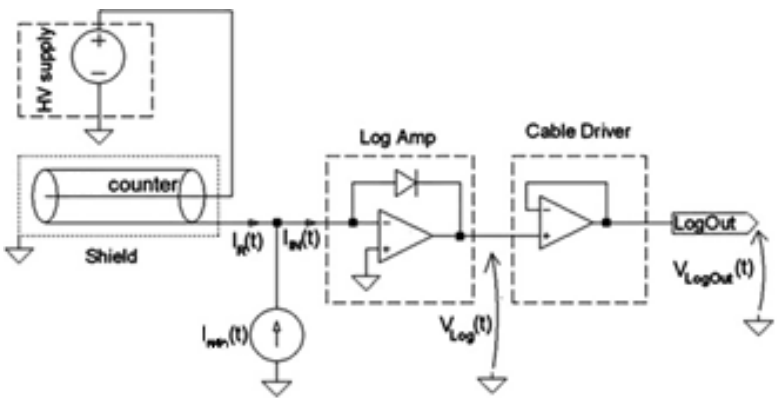

Fig 3. General scheme of the detector electronics.

A user-adjustable high voltage module on board the instrument supplies the voltage bias at the electrodes of the proportional tube. The acquisition electronics is based on a current-to-voltage logarithmic amplification of the current signal produced in the proportional counter. The choice of a logarithmic amplifier is driven by the need to measure the output current over few order of magnitude, in order to achieve the same dynamic range also in terms of burst intensity. 
From a theoretical point of view the logarithmic amplifier can accept only a positive input current. From a practical point of view, this causes that an offset current $\mathrm{I}_{\min }$ of minimum $100 \mathrm{pA}$ must be supplied in input to avoid negative saturation.

In order to provide a positive current at the input of the logarithmic amplifier the polarization of the proportional counter is positive at the anode while the cathode is grounded.

Because the signal is collected at the cathode, the proportional counter must be electrostatically shielded in order to avoid noise pick up. The electrostatic shield is made of an aluminium cylinder that completely encases the counter.

The output of the logarithmic amplifier passes through a cable driver that permits to connect long cables, up to about $200 \mathrm{~m}$.

More details about the theoretical operating principles of the circuit can be found in [7].

The output voltage of the cable driver is processed in two different ways:

1) via an oscilloscope (PicoScope442 by PicTechnology) and monitored by a LabVIEW program running on a $\mathrm{PC}$ that converts the voltage back to current.

2) Via a custom board based on a FPGA model Cyclone III produced by ALTERA.

In the oscilloscope acquisition the signal is sampled at a rate of $10 \mathrm{MHz}$. This method is mainly used to visualize the current profile in time, but is unfit for a continuous measurement because of the rather slow processing time of the software running on the PC.

In the FPGA acquisition the signal is sampled via a 12 bit $\mathrm{ADC}$ converter again at $10 \mathrm{MHz}$, but the analysis is performed on-line by the FPGA that send to a remote PC the dose value every second.

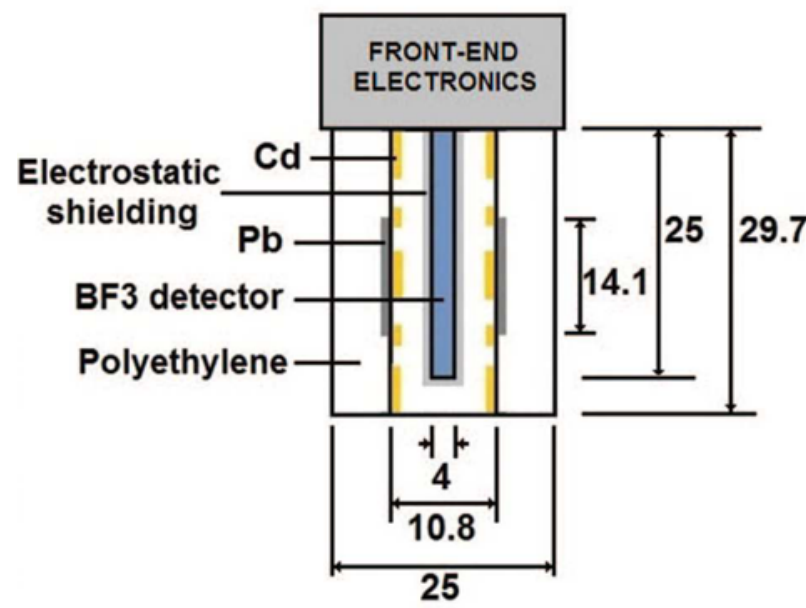

Fig 4. Sketch of the moderator of the LUPIN, measurements in $\mathrm{cm}$.

\subsubsection{Design of the moderator}

Figure 4 shows a sketch of the LUPIN moderator. It consists of a polyethylene cylinder of $25 \mathrm{~cm}$ diameter with lead and cadmium inserts. The lead layer permits to extend the energy response range up about $10 \mathrm{GeV}$ [8], while the cadmium inserts permit to tune the response to slow and thermal neutron. An electrostatic shielding, constituted by an aluminium cylinder of $1.5 \mathrm{~mm}$ thickness, contains the detector and two polyethylene inserts, which are used to fill the void around the detector.

The moderator design is optimized so that the response function of the instrument reproduces sufficiently well the curve of the conversion coefficients from neutron fluence to $\mathrm{H}^{*}(10)$ over a wide energy range, from thermal neutrons up to $10 \mathrm{GeV}$.

\subsubsection{LUPIN calibration}

In order to measure the number of neutrons interacting in the proportional counter during a neutron burst the LUPIN software integrates the current over a user settable time window and calculates the total charge generated in the proportional counter.

Dividing the integral charge by the mean collected charge (MCC) due to the interaction of a single neutron, the LUPIN can calculate the number $N$ of interacting neutrons. See equation (1)

$$
N=\operatorname{round}\left(\frac{\sum_{i=1}^{n} I_{n} \cdot \Delta t}{M C C}\right)
$$

where $N$ is the number of acquired neutrons, "round" is a function which rounds up the number in brackets to the nearest integer number, $n$ is the number of samples in a single acquisition, $I_{i}$ is the current value of each sample, $\Delta \mathrm{t}$ is the time width of the sampling (e.g. for $10 \mathrm{MHz}$ sampling, $\Delta \mathrm{t}=100 \mathrm{~ns}$ ) and $\mathrm{MCC}$ is a fundamental parameter characterizing the LUPIN.

The MCC was measured with an Am-Be source tuning the source to detector distance to obtain an interaction rate of about $10 \mathrm{~s}^{-1}$ and setting four different integration times: $0.5 \mathrm{~ms}, 1 \mathrm{~ms}, 2 \mathrm{~ms}$ and $4 \mathrm{~ms}$. The proportional counter bias voltage was $1180 \mathrm{~V}$.

Figure 5 shows the frequency distribution, the main peak corresponding to the full energy deposition of the reaction products is clearly visible. The continuum below the main peak is due to the wall effect.

The MCC corresponding to an integration time $\mathrm{t}=0.5$ $\mathrm{ms}$ is $547 \mathrm{fC}$. For higher integration times the MCC reaches a steady value of $615 \pm 3 \mathrm{fC}$, indicating that an integration time of $1 \mathrm{~ms}$ is enough to avoid any ballistic deficit.

The calibration in terms of $\mathrm{H}^{*}(10)$ has been done with a $\mathrm{Pu}-\mathrm{Be}$ source at the calibration facility at CERN and cross checked at the Politecnico di Milano with an AmBe source. The sensitivity is $2.13 \pm 0.2 \mathrm{nSv}^{-1}$.

\subsubsection{Photon rejection capability}

It is possible, especially around electron accelerators, that the radiation burst is composed by a photon and a neutron component. Usually both radiation components have a pulsed time structure. Of course also the photon component can generate charge inside the proportional counter. 


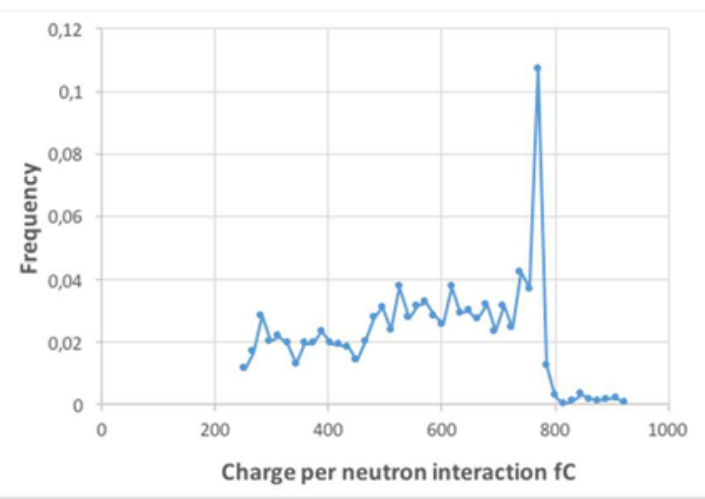

Fig 5. Frequency distribution of the charge per neutron interaction for a $4 \mathrm{~ms}$ integration time.

The separation of the two contributions can be done on a time basis considering that the photon component immediately interacts with the detector, while the interaction of the neutron component is delayed because fast neutrons take some time for the moderation and, once thermalized, a further delay due to the diffusion time is introduced.

The average thermalization and diffusion time (TDT) is typically in the $350-700 \mu$ s range [9].

Figure 6 shows an example of the exploitation of the TDT to separate the photon and neutron component. The measurement was performed at PSI around the injector test facility used as principal test and demonstration plant for the SwissFEL project. [10]

Data shown in Figure 6 was taken close to the beam dump where the photon contribution is particularly intense, compared to the neutron one.

The graph shows the typical fingerprint of a pulsed and mixed radiation field, composed by a very intense initial peak due to the photon interaction followed by neutron interactions spread over hundreds of $\mu \mathrm{s}$.

The integral charge of the photon peak is about $1100 \mathrm{fC}$, equivalent to the interaction of two neutrons, and its FWHM is around 200 ns. The gamma rejection is obtained starting the charge integration about $5 \mu$ s after the photon peak that can be used as a trigger.

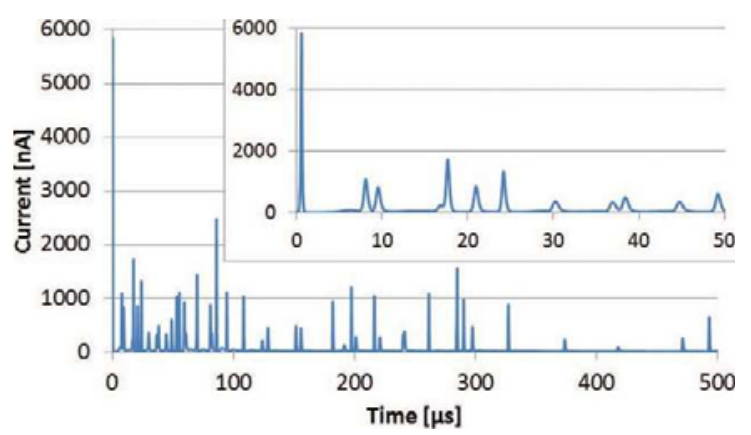

Fig 6. Current output signal for pulsed and mixed radiation fields.

\section{RESULTS AND DISCUSSION}

The results of the measurements are given in Table 1, which shows both the average $H^{*}(10)$ rate and the $H^{*}(10)$ per single burst.

The presence of a photon contribution to the neutron signal was checked for every position by sampling the signal via oscilloscope. Figure 7 shows an example of signal taken in position 5a. A comparison with figure 6 permits to conclude that the photon contribution is negligible.

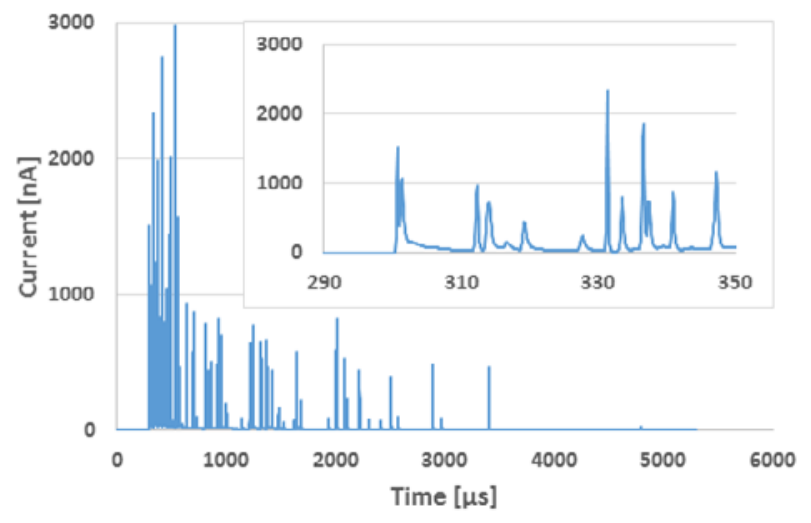

Fig 7. Current output signal measured in position 5a.

For some positions two sets of measurements were performed: the first one with ASTRID operating in slow extraction mode, the second one in fast injection mode. The high sensitivity of the device and its wide operating range allowed measuring very low average $\mathrm{H}^{*}(10)$ rate as well as comparatively high $\mathrm{H}^{*}(10)$ per burst, like the ones measured in position 5a. Here the time structure of extraction phase is composed by four different subphases, three injection sub-phases from the microtron to ASTRID and one extraction sub-phase from ASTRID to ASTRID2.

Each of the injection phase has a time duration of $800 \mathrm{~ns}$ while the extraction is over one revolution in ASTRID which is 133 ns.

Table 1. Results of the measurements. (n.a. = not applicable, $*$ $=$ fast injection mode). Position 5a reports four values of $\mathrm{H}^{*}(10)$ according to the injection-extraction time structure (see figure 8)

\begin{tabular}{|c|c|c|c|c|c|}
\hline Position & \multicolumn{4}{|c|}{$\begin{array}{l}\mathrm{H}^{*}(10) \text { per } \\
\text { burst }[\mathrm{nSv}]\end{array}$} & $\begin{array}{c}\text { Average } \\
\mathrm{H}^{*}(10) \\
\text { rate }[\mu \mathrm{Sv} / \mathrm{h}]\end{array}$ \\
\hline $1 \mathrm{a}$ & \multicolumn{4}{|c|}{5.6} & $0.39 \pm 0.11$ \\
\hline $1 b$ & \multicolumn{4}{|c|}{31.7} & $1.7 \pm 0.2$ \\
\hline 2 & \multicolumn{4}{|c|}{1.9} & $0.27 \pm 0.08$ \\
\hline 3 & \multicolumn{4}{|c|}{2.8} & $0.09 \pm 0.01$ \\
\hline $3 *$ & \multicolumn{4}{|c|}{ n.a. } & $2.6 \pm 0.8$ \\
\hline 4 & \multicolumn{4}{|c|}{ n.a. } & $0.005 \pm 0.001$ \\
\hline $4 *$ & \multicolumn{4}{|c|}{ n.a. } & $0.16 \pm 0.05$ \\
\hline $5 \mathrm{a}$ & 27.7 & 23.3 & 23.5 & 43.7 & $2.4 \pm 0.1$ \\
\hline $5 a^{*}$ & \multicolumn{4}{|c|}{ n.a. } & $422 \pm 63$ \\
\hline $5 b$ & \multicolumn{4}{|c|}{ n.a. } & $43.2 \pm 10.1$ \\
\hline
\end{tabular}


The innovative working principle of the LUPIN allowed the distinguishing of the time structure of the stray radiation field and the contribution of each subphase to the total integrated $\mathrm{H}^{*}(10)$ can be separated very well.

The average $H^{*}(10)$ per burst is given separately for each injection sub- phase and for the extraction sub-phase.

Figure 8 shows the $H^{*}(10)$ data acquired in position 5 a.

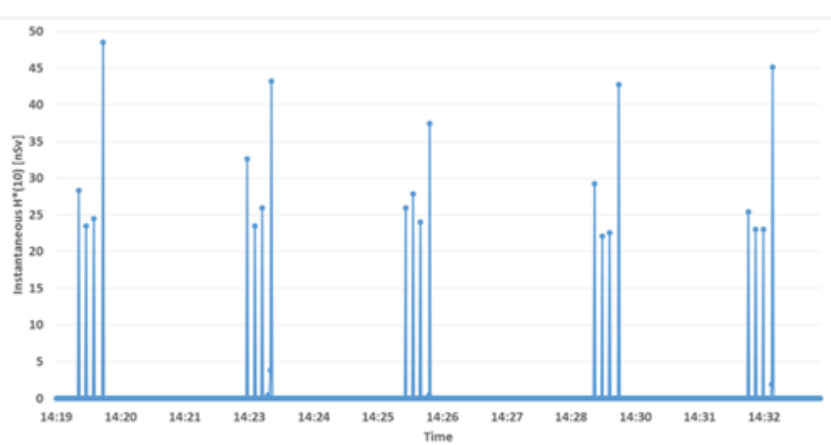

Fig 8. $H^{*}(10)$ data acquired with the LUPIN rem counter in position $5 \mathrm{a}$.

In order to cross check the consistency of the results it was performed a statistical analysis on the measurements in position $1 \mathrm{~b}$ aiming at evaluating the distribution of the bursts intensity. As the accelerator is characterized by a very high working stability, it is assumed that the fluctuation of the signal detected by the LUPIN in terms of interacting neutrons per burst is dominated by the Poisson statistic. Figure 9 shows the time profile on the number of neutron interactions recorded by the LUPIN.

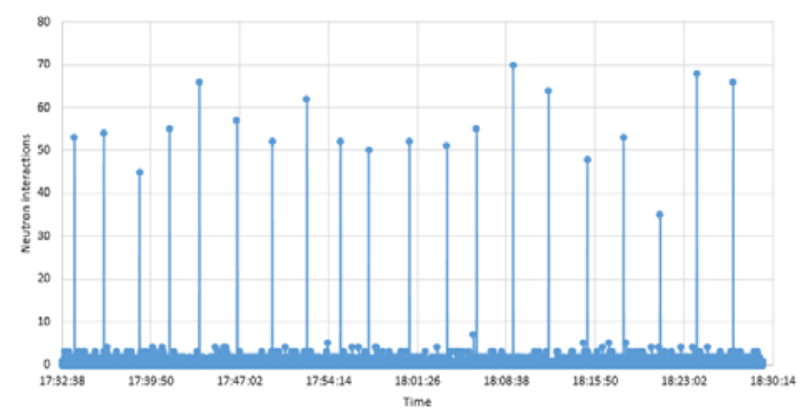

Fig 9. Data acquired with the LUPIN rem counter in position $1 \mathrm{~b}$ in terms of neutron interactions.

The injection phase from ASTRID to ASTRID2, occurring every $150-200$ s is clearly visible. Similar time distributions have been measured for the positions listed in table 2. The same table reports also the reduced $\chi^{2}$ of the distribution of the number of neutrons detected by the LUPIN during each injection, calculated assuming the Poisson distribution as target distribution.

The result is clearly shows that data are Poisson distributed with a high level of confidence. The result permits to conclude that the injection process is characterized by a very good repeatability and that the LUPIN is not affected by any signal loss.
Table 2. Statistical analysis to check the compliance to the Poisson distribution for repeated measurements in different positions. (n.a. $=$ not applicable, $*=$ fast injection mode).

\begin{tabular}{|c|c|c|c|}
\hline Position & $\begin{array}{c}\text { Reduced } \\
\chi^{2}\end{array}$ & $\begin{array}{c}\text { Degree } \\
\text { of } \\
\text { freedom }\end{array}$ & $\begin{array}{c}90 \% \\
\text { confidence } \\
\text { limits }\end{array}$ \\
\hline $1 \mathrm{a}$ & 0.991 & 22 & $0.53-1.58$ \\
\hline $1 \mathrm{~b}$ & 1,05 & 19 & $0.53-1.59$ \\
\hline 2 & 1,09 & 22 & $0.53-1.58$ \\
\hline 3 & 0.985 & 25 & $0.58-1.51$ \\
\hline $3^{*}$ & 1,09 & 450 & $0.89-1.11$ \\
\hline 4 & 0.997 & 4750 & $0.96-1.03$ \\
\hline $4^{*}$ & 1.15 & 150 & $0.82-1.2$ \\
\hline $\begin{array}{c}5 \mathrm{a} \\
\text { injection } 1\end{array}$ & 0.62 & 4 & $0.18-2.37$ \\
\hline $\begin{array}{c}5 \mathrm{a} \\
\text { injection } 2\end{array}$ & 0.49 & 4 & $0.18-2.37$ \\
\hline $\begin{array}{c}5 \mathrm{a} \\
\text { injection 3 }\end{array}$ & 0.15 & 4 & $0.18-2.37$ \\
\hline $\begin{array}{c}5 \mathrm{a} \\
\text { extraction }\end{array}$ & 0.88 & 4 & $0.18-2.37$ \\
\hline $5 \mathrm{a} *$ & n.a. & n.a. & n.a. \\
\hline $5 \mathrm{~b}$ & 1.08 & 79 & $0.75-1.27$ \\
\hline
\end{tabular}

The value of the reduced $\chi^{2}$ for position $5 \mathrm{a} *$ (fast injection mode) has not been reported as the signal has a time distribution as reported in figure 10. The fast injection mode produces an almost continuous neutron stray field with superimposed peaks due to the extraction form ASTRID to ASTRID2, occurring every $12 \mathrm{~s}$. Of course this measurement cannot be Poisson distributed. However considering just the number of neutron interactions in the peak, a value of $\chi^{2}$ (reduced) $=1.29$ is obtained, with a number of degree of freedom $v=19$. This value is within the statistical limit. (see table 2)

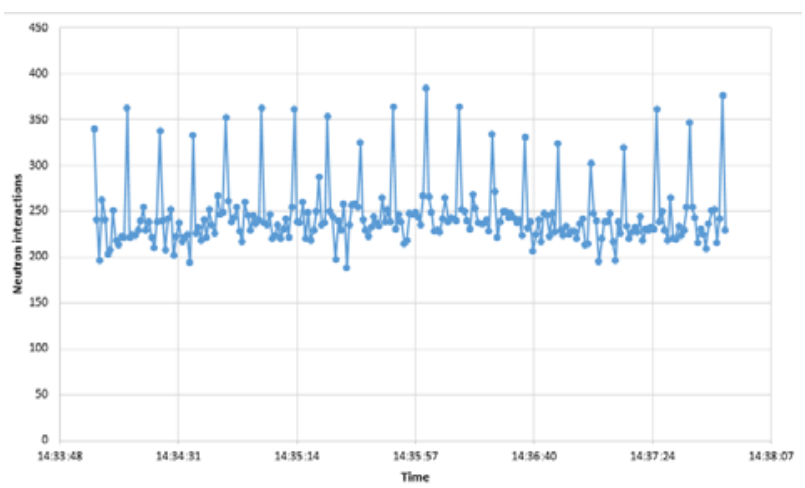

Fig 10. Data acquired with the LUPIN rem counter in position $5 \mathrm{a}$ in terms of neutron interactions. Accelerator operating in fast injection mode.

\section{Conclusion}

The measurement campaign performed with the ELSE NUCLEAR LUPIN 5401 BF3-NP rem counter at ISA allowed characterising the neutron $\mathrm{H}^{*}(10)$ rate in selected 
positions around the ASTRID and ASTRID2 storage rings.

The stray field was characterised by intense pulsed bursts of radiation, in the range of $20-50 \mathrm{nSv}$. In a previous work of benchmark of rem counters in pulsed neutron field [1], it was demonstrated that in this burst intensity range almost all the rem counters are affected by important signal loss (up to a factor 5). The LUPIN is an exception because, thanks to the front end electronics, the linearity extends up to hundreds of nSv per burst.

The radiation fields around the ASTRID accelerator complex, in spite of the very high instantaneous dose rate, results in a pretty low average dose rate, in the range of few $\mu \mathrm{Sv} / \mathrm{h}$. This means that these areas can be attended by workers of even general public (In position $1 \mathrm{~b}$ there is the visitor platform). As a consequence of that that, environmental dosimetry plays a key role in the radiation protection and the need of a specific device capable to cope with pulsed neutron fields is even more pressing.

The pulsed photon component associated to the stray neutron field was negligible, in the sense that its intensity that cannot affect the neutron measurements.

The particular time structure of the stray radiation field defined by the working parameters of the accelerators is coherent with the measurement and the statistical distribution of repeated measurement is in agreement with the Poisson distribution.

\section{References}

1. M. Caresana et al., NIM A 737, 203-213 (2014)

2. M. Caresana, M. Ferrarini, G.P. Manessi, M. Silari and V. Varoli, NIM A, 712, 15-26 (2013).

3. M. Caresana, C. Cassell, M. Ferrarini, E. Hohmann, G.P. Manessi, S. Mayer, M. Silari and V. Varoli, Rev Sci Instrum, 85, 065102 (2014).

4. J.S. Nielsen, N. Hertel and S.P. Moller, Proceedings of IPAC2011, San Sebastian, Spain, THPC003, 2909-2911 (2011).

5. N. Hertel, S. Vrønning Hoffmann, 24-1, (2011)

6. A. Klett and A. Leuschner, in Proceedings of the IEEE 2006 Nuclear Science Symposium and Medical Imaging Conference, San Diego, CA, 29

7. M. Ferrarini, V. Varoli, A. Favalli, M. Caresana, B. Pedersen, NIM A, 613, 272 (2010).

8. C. Birattari, A. Esposito, A. Ferrari, M. Pelliccioni, T. Rancati, M. Silari, Radiat Prot Dosim, 76, 135 (1998)

9. A. L. Justus, Health Phys, 102, 9 (2012)

10. M. Pedrozzi, SwissFEL Injector Conceptual Design Report, PSI Report, 10-05, (2010) 